\title{
A new technique to identify arbitrarily shaped noise sources
}

\author{
Roberto A. Tenenbaum ${ }^{\mathrm{a}}$ and Marcelo Bruno S. Magalhães ${ }^{\mathrm{b}}$ \\ ${ }^{a}$ Instituto Politécnico do Rio de Janeiro, IPRJ/UERJ, Rua Alberto Rangel, s/n, Vila Nova, 28630-050, Nova \\ Friburgo, RJ, Brazil \\ Tel.: +55 (22) 25288545 poste 315; Fax: +55 (22) 2528 8536; E-mail: tenenbaum@iprj.uerj.br \\ ${ }^{\mathrm{b}}$ FSAO Virtual Series Department, Ford Motor Company \\ E-mail: mmagalh4@ford.com
}

\begin{abstract}
Acoustic intensity is one of the available tools for evaluating sound radiation from vibrating bodies. Active intensity may, in some situations, not give a faithful insight about how much energy is in fact carried into the far field. It was then proposed a new parameter, the supersonic acoustic intensity, which takes into account only the intensity generated by components having a smaller wavenumber than the acoustic one. However, the method is only efective for simple sources, such as plane plates, cylinders and spheres. This work presents a new technique, based on the Boundary Elements Method and the Singular Value Decomposition, to compute the supersonic acoustic intensity for arbitrarily shaped sources. The technique is based in the Kirchoff-Helmholtz equation in a discretized approach, leading to a radiation operator that relates the normal velocity on the source's surface mesh with the pressure at grid points located in the field. Then, the singular value decomposition technique is set to the radiation operator and a cutoff criterion is applied to remove non propagating components. Some numerical examples are presented.
\end{abstract}

Keywords: Supersonic intensity, vibroacoustics, identification, noise control, Acoustics

\section{Introduction}

Acoustic radiation is a broadly covered subject in the literature. One of its branches is the so-called source localization, or backpropagation problem. Since the first published works, like those of Stepanishen and Benjamim [14], Maynard et al. [9] and Sarkissian [13], among others, the existence of an important source of error, caused by the existence of evanescent waves present in the spatial spectrum of the sound pressure is mentioned and some methods like the wavenumber domain filtering (see Maynard et al. [9]) were proposed to decrease their influences on the backpropagated field. The evanescent waves do not have any significant contribution to the overall sound power, since their influence decays in an exponential fashion with the distance to the source. If, instead of the backpropagation problem, one is interested in understanding how a particular source generates sound power, an interesting approach would be to somehow filter the source normal velocity so that the part of its spatial spectrum that produces evanescent waves is deleted. The truncated spectrum that is left after the suppression will then contain only components that do contribute to sound power. This idea, first outlined by Borgiotti [1], Borgiotti and Rosen [3], and Borgiotti and Jones [2], is the base from which the concept of supersonic intensity, presented by Williams [16,17], is developed.

Acoustic intensity is the most usually employed quantity when sound radiation analysis is concerned. A trap is however hidden behind the frequencies lower than a local coincidence criterion. Williams [18], based on a spatial Fourier analysis of the sound field generated by cylindrical radiators, shows that the interaction between components with wavelength shorter than the acoustic one (called subsonic components), which generate evanescent waves, can indeed generate a not negligible amount of active intensity. The strategy proposed is then to discard the influence of those components so that a new quantity, referred to as supersonic acoustic intensity, that faithfully describes 
how much energy is in fact transported to the far-field, can be extracted either from sound intensity measurements or numerical simulation. In a later work, Williams [17] details of the evaluation of supersonic acoustic intensity for planar radiators, showing the relationship between the interaction between supersonic components and the active intensity are given.

The wavenumber domain approach used by Williams $[16,17]$ is, however, not applicable in cases where the source geometry is not separable in any suitable coordinate system. For these cases, an alternative approach, whose some of the initial ideas were outlined by Williams [19], is then to use the Kirchoff-Helmholtz integral equation [10,12] to obtain a radiation operator relating the source normal vibration velocity and the sound pressure field. In this work, the Kirchoff-Helmholtz equation is discretized so as to allow the representation of the radiation operator as a matrix which, in its turn, represents a linear transform between the vector of source normal velocity values at some points and the pressure on a set of grid points located in the fluid medium that surrounds the source. The basic idea is to discard the lowest singular values present in the singular value decomposition (SVD) of the radiation operator, which are well-known $[1,2,10,14]$ to be related to very inefficient radiation modes.

Some interesting questions arise when the Kirchoff-Helmholtz equation is used. In this case, for instance, there is not a clear distinction between propagating and non propagating components. This can cause significant errors in the evaluation of the generalized supersonic intensity if a smart cutoff criterion is not used. This work presents a proposal of such a criterion based on the convergence of the sound power with respect to the number of singular values taken into account in the supersonic intensity evaluation.

\section{Wavenumber domain approach}

In the case the sound source geometry is separable in a suitable coordinates system, the most adequate approach is the one based on the spatial Fourier transform, also known as the wavenumber domain approach $[9,13,19]$. The basic idea is to use the spatially Fourier-transformed versions of both sound pressure and velocity on the surface of the source. In the sequel, for the sake of simplicity, only the case of a planar source lying on the plane $z=0$ will be dealt with. In this case, the pressure and velocity can be written as

$$
\begin{aligned}
& \hat{p}(x, y, 0, \omega)=\int_{-\infty}^{\infty} \int_{-\infty}^{\infty} \tilde{p}\left(k_{x}, k_{y}, 0, \omega\right) e^{i\left(k_{x} x+k_{y} y\right)} d k_{x} d k_{y}, \\
& \hat{\mathbf{v}}(x, y, 0, \omega)=\int_{-\infty}^{\infty} \int_{-\infty}^{\infty} \tilde{\mathbf{v}}\left(k_{x}, k_{y}, 0, \omega\right) e^{i\left(k_{x} x+k_{y} y\right)} d k_{x} d k_{y},
\end{aligned}
$$

where $\hat{p}$ is the pressure in the frequency domain and $\tilde{p}$ is the pressure in the wavenumber domain, also called the angular spectrum of $\hat{p} ; \hat{\mathbf{v}}$ and $\tilde{\mathbf{v}}$, in the same manner, represent the velocity in frequency and wave-number domains, with $k_{x}$ and $k_{y}$ being the wave-numbers in $x$ and $y$ directions. If one assumes that the planar source is located on the plane $z=0$, the relationship between the angular spectra of the pressure at any given plane parallel to the source and the angular spectrum of its normal velocity is given by Williams [18,20]

$$
\tilde{p}\left(k_{x}, k_{y}, z, \omega\right)=\frac{\rho_{0} c k \tilde{v}_{n}}{k_{z}} e^{i k_{z} z},
$$

where $k_{z}^{2}=k^{2}-k_{x}^{2}-k_{y}^{2}$ and $\tilde{v}_{n}$ is the angular spectrum of the velocity component on the normal direction $(z)$. In order to obtain the frequency spectrum of the pressure field one can then use the inverse spatial Fourier transform,

$$
\hat{p}(x, y, z, \omega)=\mathcal{F}^{-1}\left[\frac{\rho_{0} c k \tilde{v}_{n}}{k_{z}} e^{i k_{z} z}\right],
$$

where the operator $\mathcal{F}^{-1}$ denotes the inverse Fourier transform. If one wants to eliminate those wavelengths that originate evanescent waves, no $\left(k_{x}, k_{y}\right)$ pair lying outside the radiation circle $S_{r}$ defined by $k_{x}^{2}+k_{y}^{2}<k^{2}$ should be taken into account during sound intensity evaluation. The strategy proposed by Williams [16] is to perform the integrations contained in Eqs (1)-(2) only within the radiation circle, therefore obtaining modified versions of pressure and velocity on the source surface, containing only radiating (supersonic) wavelengths. These versions, 
termed supersonic velocity and supersonic pressure are then defined as

$$
\begin{aligned}
& \hat{p}^{(s)}(x, y, 0, \omega)=\int_{S_{r}} \int_{S_{r}} \tilde{p}\left(k_{x}, k_{y}, 0, \omega\right) e^{i\left(k_{x} x+k_{y} y\right)} d k_{x} d k_{y}, \\
& \hat{\mathbf{v}}^{(s)}(x, y, 0, \omega)=\int_{S_{r}} \int_{S_{r}} \tilde{\mathbf{v}}\left(k_{x}, k_{y}, 0, \omega\right) e^{i\left(k_{x} x+k_{y} y\right)} d k_{x} d k_{y},
\end{aligned}
$$

with the superscript $(s)$ denoting supersonic quantities. It is now possible to obtain the so-called supersonic intensity by multiplying the supersonic pressure by the complex conjugate of the supersonic velocity, which yields

$$
\hat{\mathbf{I}}^{(s)}(x, y, 0, \omega)=\frac{1}{2} \operatorname{Re}\left[\hat{p}^{(s)}(x, y, 0, \omega) \hat{\mathbf{v}}^{*(s)}(x, y, 0, \omega)\right] .
$$

It can be shown $[16,18]$ that the sound power obtained by integrating the supersonic intensity over the entire source surface is equal to the one obtained by doing the same process with the conventional active intensity.

\section{Kirchoff-Helmholtz equation approach}

Let $\Omega$ be the fluid domain in which the source, whose interior is denoted by $\Omega_{i}$, is immerse, $\mathbf{x}_{s}$ an arbitrary point on its boundary $\Gamma$ and $\mathrm{x}$ an arbitrary point in $\Omega$. The relationship between the normal velocity and the acoustic pressure, in the frequency domain, is given by $[4,11]$

$$
c \hat{p}(\mathbf{x}, \omega)=\int_{\Gamma}\left(i \omega \rho_{0} \hat{v}_{n}\left(\mathbf{x}_{\mathbf{S}}, \omega\right) G\left(\mathbf{x}_{s} \mid \mathbf{x}\right)-\hat{p}\left(\mathbf{x}_{\mathbf{S}}, \omega\right) \frac{\partial G\left(\mathbf{x}_{s} \mid \mathbf{x}\right)}{\partial \mathbf{n}_{s}}\right) d \mathbf{x}_{s}
$$

with $c=0$ for $\mathbf{x} \in \Omega_{i}, c=1$ for $\mathbf{x} \in \Omega$, and $c=\alpha / 4 \pi$ for $\mathbf{x} \in \Gamma$, where $\Gamma$ is the source boundary, whose inward-pointing normal is $\mathbf{n}_{s}, \omega$ is the angular frequency, $\rho_{0}$ is the fluid density, $\hat{v}_{n}$ is the normal component of the source boundary velocity, $\hat{p}$ is the acoustic pressure at an arbitrary point and $\alpha$ the solid angle defined by any non-smoothness that may exist on $\Gamma$, that is, if the boundary is smooth, then $\alpha=2 \pi$ and $c=0.5 . G\left(\mathbf{x}_{s} \mid \mathbf{x}\right)$ is the free-field Green function, given by

$$
G\left(\mathbf{x}_{s} \mid \mathbf{x}\right)=\frac{e^{i k\left|\mathbf{x}_{s}-\mathbf{x}\right|}}{4 \pi\left|\mathbf{x}_{s}-\mathbf{x}\right|}
$$

It is important to notice that, in order to obtain the sound pressure at any point located in $\Omega$, one must first evaluate it on the source boundary $\Gamma$, that is, Eq. (8) must be solved with $c=0.5$ (assuming a smooth boundary). Only after that can Eq. (8) be solved with $c=1$. If $\Gamma$ is discretized using boundary elements, these steps can be approached as matrix operations. While solving for the pressure on $\Gamma$ one has,

$$
\mathbf{H} \hat{\mathbf{p}}=\mathbf{G} \hat{\mathbf{v}},
$$

where $\mathbf{H}$ is a matrix containing integrals of the product of the Green function normal derivatives by each of the boundary elements interpolation functions, $\hat{\mathbf{p}}$ is a vector containing pressure values at each of the boundary nodes, $\mathbf{G}$ is a matrix containing integrals of the product of the Green function by the elements interpolation functions and $\hat{\mathbf{v}}$ is a vector containing values of the normal component of the boundary velocity [4,21]. Once Eq. (10) is solved for $\hat{\mathbf{p}}$, the pressure anywhere on $\Omega$ can be obtained using the discretized version of Eq. (8),

$$
\hat{\mathbf{p}}_{\Omega}=\mathcal{G} \hat{\mathbf{v}}-\mathcal{H} \hat{\mathbf{p}}
$$

where the matrices $\mathcal{G}$ and $\mathcal{H}$ have the same structure of matrices $\mathbf{G}$ and $\mathbf{H}$, with the difference that the Green functions are now evaluated between points in $\Omega$ and points on $\Gamma$. $\hat{\mathbf{p}}_{\Omega}$ is a vector containing pressure values at the desired points in $\Omega$. Replacing Eq. (10) in Eq. (11) one obtains an expression that relates directly the source boundary vibration velocity to the sound pressure at the field points in $\Omega$,

$$
\hat{\mathbf{p}}_{\Omega}=\left(\mathcal{G}-\mathcal{H} \mathbf{H}^{-1} \mathbf{G}\right) \hat{\mathbf{v}} .
$$

The term $\left(\mathcal{G}-\mathcal{H} \mathbf{H}^{-1} \mathbf{G}\right)$ can be though of as being a radiation operator in discretized form and will be denoted in the sequel as $\mathcal{R}$. In the next section an approach will be described in order to dissect the radiation operator and identify the components which do inject a significant amount of energy into the far-field. 


\section{Supersonic intensity for abitrarily shaped sources}

The basic idea for evaluating the supersonic intensity for arbitrarily shaped sources is to use the singular value decomposition (SVD) [1,5] of the radiation operator, that is, to rewrite it as

$$
\mathcal{R}=\mathbf{U} \Sigma \mathbf{V}^{H}
$$

where $\mathbf{U}$ is a $M \times M$ matrix, $\Sigma$ is a $M \times N$ diagonal matrix whose elements, always non-negative and decreasingly ordered, are referred to as the singular values of $\mathcal{R}$ and $\mathbf{V}$ is a $N \times N$ matrix. The superscript $H$ denotes conjugate transpose. Some of the features of the SVD are of great importance for its application on sound radiation problems. For instance, the columns of $\mathbf{V}$ form an orthonormal basis for the domain of $\mathcal{R}$, while the columns of $\mathbf{U}$ span the column space of $\mathcal{R}$. The columns of $\mathbf{V}$ are termed by Borgiotti [1] singular velocity patterns.

The singular values of $\mathcal{R}$ play an important role in the structure of the radiation operator. Writing Eq. (13) in an alternative form [1]

$$
\mathcal{R}=\sum_{i=1}^{N} \sigma_{i} U_{i} V_{i}^{H},
$$

where $U_{i}$ are the columns of $\mathbf{U}$ and $V_{i}^{H}$ the lines of $\mathbf{V}^{H}$, it is noticed that the $i$-th singular value $\sigma_{i}$ multiplies the $i$-th line of $\mathbf{V}^{H}$, which, in its turn, corresponds to the $i$-th singular velocity pattern. Replacing Eq. (14) into Eq. (12) one obtains

$$
\hat{\mathbf{p}}_{\Omega}=\sum_{i=1}^{N} \sigma_{i} U_{i}<V_{i}^{H}, \hat{\mathbf{v}}>.
$$

Equation (15) shows that if $\sigma_{i}$ is too small, the contribution of the $i$-th singular velocity pattern to the pressure field will also be too small. The singular value corresponding to a given singular velocity pattern can therefore be used as an estimate of its radiation efficiency. This analogy between radiation efficiency and singular values allows the use of the SVD to separate the patterns with high efficiency (the "radiating" ones) from those with low efficiency (the "evanescent" ones) by looking at the singular value associated with each one. It is important to notice that when using the SVD approach, the patterns associated with low singular values are not actually evanescent, since they still have a small contribution to the sound power. If a limit $\sigma_{l}$ is set as the cutoff singular value, the singular velocity patterns $\mathbf{V}_{i}$ with $i>l$ can be discarded without causing any significant effect to the sound power value. Indeed, let $c_{i}$ be the projection of the source boundary normal velocity onto the $i$-th singular velocity pattern,

$$
c_{i}=\mathbf{V}_{i} \cdot \hat{\mathbf{v}} .
$$

Borgiotti [1] shows that the sound power is proportional to the series

$$
\Pi=\sum_{i=1}^{N} \sigma_{i}^{2}\left|c_{i}\right|^{2},
$$

with $\Pi$ being referred to as non-dimensional power and which, if truncated at $i=i_{l}$ with $l<N$ and provided the discarded singular values are neglectable, will be a good approximation, showing that those values do not have a significant contribution to sound power.

One is now able to obtain the generalized supersonic velocity $\hat{\mathbf{v}}^{(s)}$ from the retained singular velocity patterns by using the orthonormality property of the columns of $\mathbf{V}$, that is,

$$
\hat{\mathbf{v}}^{(s)}=\sum_{i=1}^{l}\left(\mathbf{V}_{i} \cdot \hat{\mathbf{v}}\right) \hat{\mathbf{v}} .
$$

The supersonic acoustic pressure on the source surface can, on the other hand, be obtained using Eq. (10), being given by

$$
\hat{\mathbf{p}}^{(s)}=\mathbf{H}^{-1} \mathbf{G} \hat{\mathbf{v}}^{(s)} .
$$


The component normal to the source boundary of the supersonic intensity is obtained using

$$
\mathcal{I}_{n_{i}}^{(s)}=\frac{1}{2} \Re\left(\hat{p}_{i}^{(s)} \hat{v}_{i}^{(s)^{*}}\right),
$$

where $\hat{p}_{i}^{(s)}$ and $\hat{v}_{i}^{(s)^{*}}$ denote, respectively, the pressure and the velocity conjugate on the $i$-th node of the source boundary mesh and $\Re$ stands for real part.

Taking the SVD of the radiation operator, one has

$$
\hat{\mathbf{p}}_{\Omega}=\mathbf{U} \Sigma \mathbf{V}^{H} \hat{\mathbf{v}}
$$

Comparing Eq. (21) with Eq. (4) and using the orthogonality properties of $\mathbf{U}$ and $\mathbf{V}$ some of those analogies can be identified. The multiplication of matrix $\mathbf{V}^{H}$ by the velocity $\hat{v}_{n}$ is similar to performing the spatial Fourier transform which leads to $\tilde{v}_{n}$ in Eq. (4) since it also denotes a projection of $\hat{v}_{n}$ onto an orthonormal basis, the columns of $\mathbf{V}$. Multiplication by matrix $\Sigma$ corresponds to the multiplication of $\tilde{v}_{n}$ by the term $\rho_{0} c k e^{i k_{z} z} / k_{z}$, since it is related to the radiation efficiency of each one of the basis vectors onto which the velocity is projected. At last, multiplication by matrix $\mathbf{U}$ corresponds to the inverse spatial Fourier transform, which is necessary for obtaining the sound pressure at the field points.

The choice of an optimum value for $l$ in Eq. (18) is, of course, of great importance. If too small a value is chosen, an excessive quantity of singular values is discarded, causing the supersonic intensity not to take into account singular velocity patterns which do contribute to the sound power. On the other hand, if too large a value is used, singular velocity patterns which generate a negligible amount of sound power will also be taken into account in the supersonic velocity evaluation. In the former case, the sound power obtained by the integration of the supersonic intensity over the source boundary will be smaller than its actual value, while in the latter, although the sound power so calculated is very close to the actual one, the supersonic intensity will be masked by the presence of nopropagating patterns, impairing its feature of discarding hotspots that are actually caused by energy recirculation. It is however possible to establish a criterion for an automatic choice of $i_{l}$, based on the sound power. The idea is to closely monitor the value obtained by Eq. (17) as each term in the summation is added. When the addition of new terms produces a change in the sound power smaller than a preset limit $\delta$, that is,

$$
\epsilon=\frac{\sum_{i=1}^{(l+1)} \sigma_{i}^{2}\left|c_{i}\right|^{2}-\sum_{i=1}^{l} \sigma_{i}^{2}\left|c_{i}\right|^{2}}{\sum_{i=1}^{l} \sigma_{i}^{2}\left|c_{i}\right|^{2}}<\delta,
$$

the current amount of terms is considered to be the ideal number of singular velocity patterns to be retained in order not to include the nonpropagating ones.

\section{Numerical examples}

The generalized supersonic intensity values should converge to the conventional active intensity as the frequency increases. In particular, if a local coincidence frequency can be identified, then both kinds of intensity should be equal for frequencies higher than that.

Let us consider a flat-capped cylinder vibrating in a $(6,2)$ mode, that is, having six anti-nodes in the axial direction and two in the tangential one (see Fig. 1).

The coincidence frequency $f_{c}$ is defined to be the one which provides a wavelength that is equal to the distance between two adjacent anti-nodes, which yields in this case $f_{c}=233 \mathrm{~Hz}$. A non dimensional frequency, defined as $\nu=f / f_{c}$ was then varied from 0.6 to 1.0. This range is enough to show the effect of canceling on the discrepancy observed between the generalized supersonic intensity and the conventional active intensity. Figures 2 to 5 show the two intensities computed for $\nu=0.6$ and $\nu=1$ (see Magalhães, Tenenbaum and Zindeluk [7] and Magalh ães [6]).

The results show that, in fact, as the frequency approaches coincidence, the generalized supersonic intensity converges to the conventional active intensity, as well as the radiation efficiency tends to unity, what is an expected result, since the cancellation mechanism gets more and more negligible as the frequency increases. 


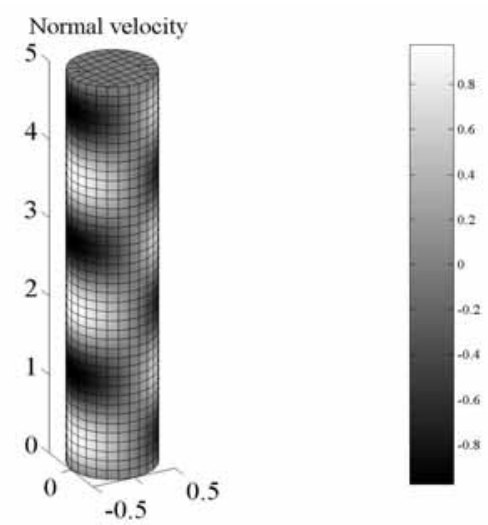

Fig. 1. Vibration mode used in the convergence study.

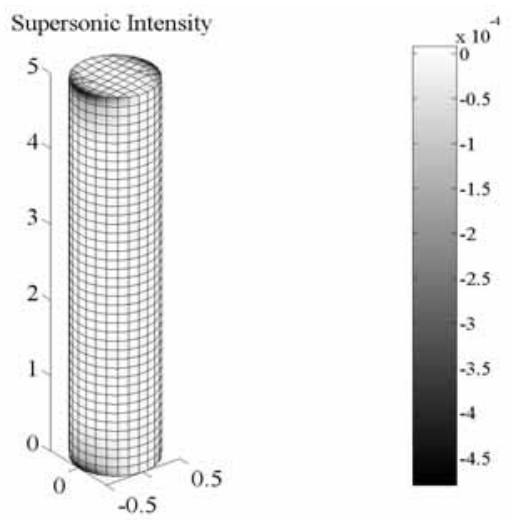

Fig. 2. Supersonic intensity $\left(\mathrm{W} / \mathrm{m}^{2}\right), \nu=0.6$.

\section{Corner and edge modes identification}

The relation between the spatial distribution of anti-nodes at a given vibration mode and the acoustic wavelength is of great importance in determining if the mode will behave as and edge-mode, a corner-mode or a surfacemode [8]. The generalized supersonic intensity should be capable of identifying these modes, since it eliminates the recirculation patterns generated by the cancelation between adjacent anti-nodes below coincidence. Although this mode classification is not directly applicable for arbitrarily shaped sources, a numerical test was conducted with the same flat-capped cylinder used in the previous section. That geometry, in spite of being not separable, is significantly regular so that its vibration modes still can be roughly classified with respect to their acoustic behavior. In the test conducted in this section there can be no corner modes, since, of course, the source has no edge in the circumferential direction. Therefore, only edge and surface modes can be detected. The test consists of evaluating both the generalized supersonic and conventional active intensity for the $(6,0)$ mode which has six anti-nodes in the axial direction and none in the tangential one, that is, the anti-nodes behave as ring sources. The coincidence frequency in this case is $205.8 \mathrm{~Hz}$.

At a non-dimensional frequency $\nu=0.5$, the mode $(6,0)$ behaves like an edge mode, radiating almost only by the lower and upper boundary of the cylinder. This configuration, as shown in Fig. 6, is identified by the generalized supersonic intensity evaluated on the source boundary, where the higher values in the neighborhood of the caps are clearly noticeable. It is worth noticing that, since during the calculation of the radiation operator, an inward normal was used, negative intensity values mean actually outbound radiation. The conventional active intensity, as shown in 

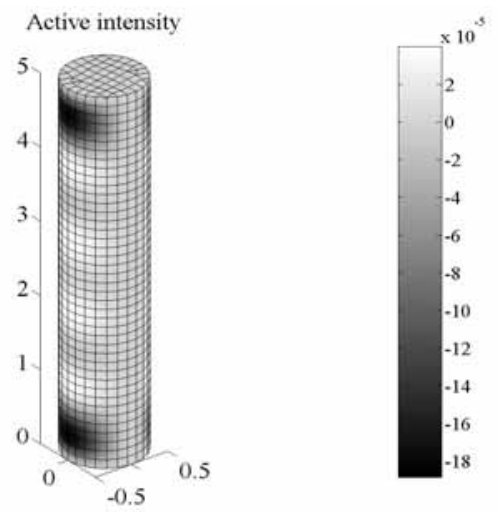

Fig. 3. Active intensity $\left(\mathrm{W} / \mathrm{m}^{2}\right), \nu=0.6$.
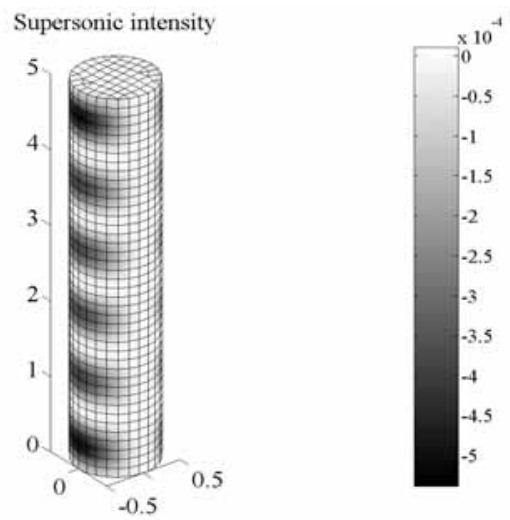

Fig. 4. Supersonic intensity $\left(\mathrm{W} / \mathrm{m}^{2}\right), \nu=1.0$.
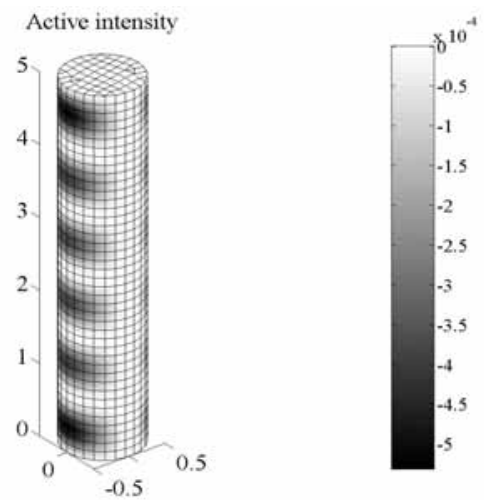

Fig. 5. Active intensity $\left(\mathrm{W} / \mathrm{m}^{2}\right), \nu=1.0$.

Fig. 7, on the other hand, shows also the regions where there is a significant amount of recirculation due to canceling, leading to some confusion about which areas actually contribute to the sound power.

A second numerical test was conducted using a source with a shape of a simplified outer shell of a combustion engine with 4 in-line cylinders. In this particular case, the radiation was studied at $40 \mathrm{~Hz}$. This frequency corresponds 

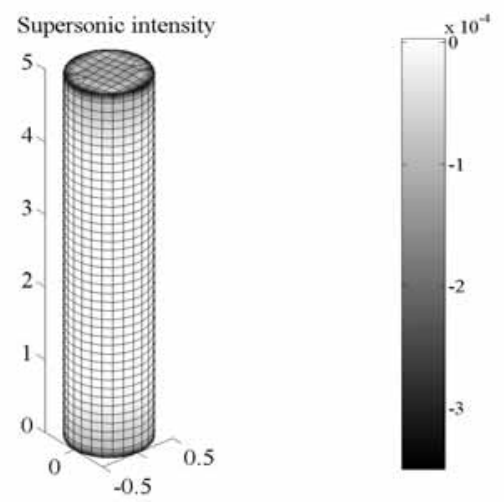

Fig. 6. Supersonic intensity $\left(\mathrm{W} / \mathrm{m}^{2}\right), \nu=0.5$.

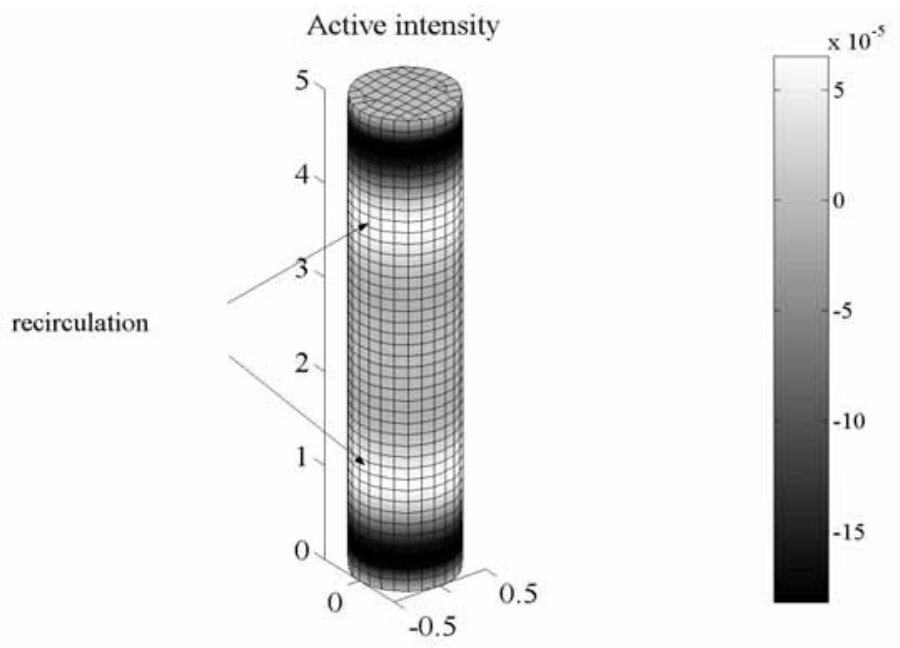

Fig. 7. Active intensity $\left(\mathrm{W} / \mathrm{m}^{2}\right), \nu=0.5$.

to the main combustion loads found at typical engine idle speeds $(\approx 1200 \mathrm{rpm})$.

Figures 8 and 9 show that some active intensity peaks on the source surface are created, actually, by energy recirculation patterns, since they do not appear on the supersonic intensity distribution. Figure 9 shows also that the regions close to the edges are the main contributors to the overall sound power.

\section{Singular participation}

The analysis of other quantities that are calculated during the process of obtaining the supersonic intensity can give further insight about the radiation of a given source. The coefficients $c_{i}$ in Eq. (17) are, as mentioned before, a projection of the normal velocity over the singular velocity patterns, being therefore a kind of modal participation of the normal velocity. Since the basis used for the projection is not the modal one, but is instead formed by the singular velocity patterns, the vector formed by the coefficients $c_{i}$ will be referred to as the singular participation. These coefficients, when analyzed together with the decay curve of the radiation operator singular values and the convergence curve of the sound power, furnish a tool for identifying which velocity patterns radiate with high efficiency into the far field and are, therefore, taken into account when the supersonic intensity is built. Besides, the analysis of the singular participation curve can also be used to evaluate the relative importance of each one of them. 


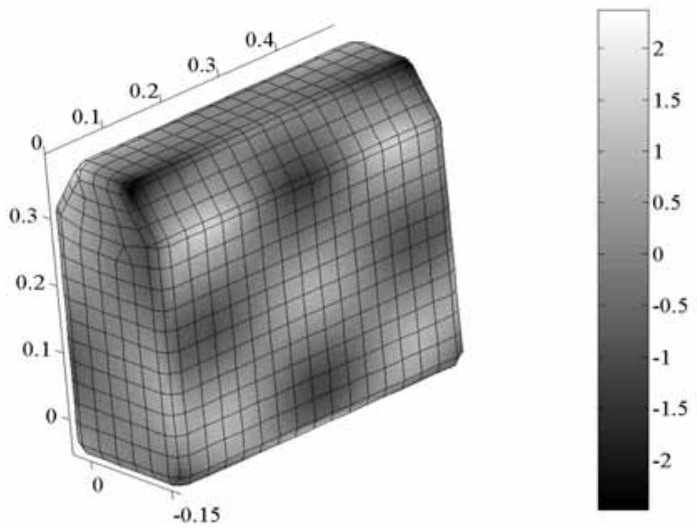

Fig. 8. Active intensity $\left(\mathrm{W} / \mathrm{m}^{2}\right), f=40 \mathrm{~Hz}$.

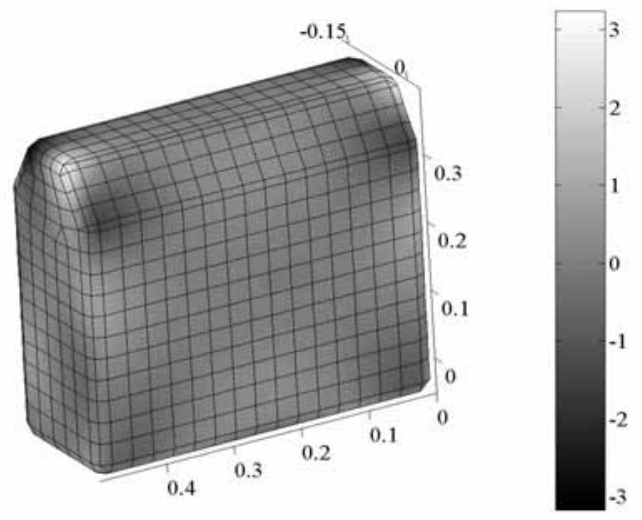

Fig. 9. Supersonic intensity $\left(\mathrm{W} / \mathrm{m}^{2}\right), f=40 \mathrm{~Hz}$.

Figures 10 to 13 show, respectively, the singular participation, singular values decay curve and sound power convergence curve for the flat-capped cylinder vibration in the $(6,0)$ mode.

Figure 10 shows that the normal velocity at $\nu=0.5$ is built basically by using four singular velocity patterns, since the singular participation curve is virtually null, except for the four peaks. The dashed part of the curve denotes the singular velocity patterns that were taken into account for calculating the supersonic intensity. The fact that only a small part of the curve was used can be justified by looking at Fig. 13, where the convergence of the sound power as a function of the number of singular velocity patterns retained is plotted. As can be noticed, as soon as the velocity pattern corresponding to the first peak in Fig. 10 is added, the sound power reaches its final value, showing that it receives virtually no contribution from the peaks corresponding to other velocity patterns, which can then be discarded for evaluating the supersonic intensity. The singular values decay curve of the radiation operator can give further insight to the interpretation of the sound power convergence curve. Looking together to Figs 10 and 11, it can be noticed that the first peak in the singular participation occurs for the singular velocity pattern corresponding to the $10^{\text {th }}$ singular value. At the decay curve, it can be seen that it corresponds to about $20 \%$ of the first singular value $\left(\sigma_{1}\right)$, meaning that it has a fairly high radiation efficiency. On the other hand, the second peak on the singular participation curve corresponds to the 30th singular velocity pattern, whose singular value is about $0.1 \%$ of $\sigma_{1}$, that 


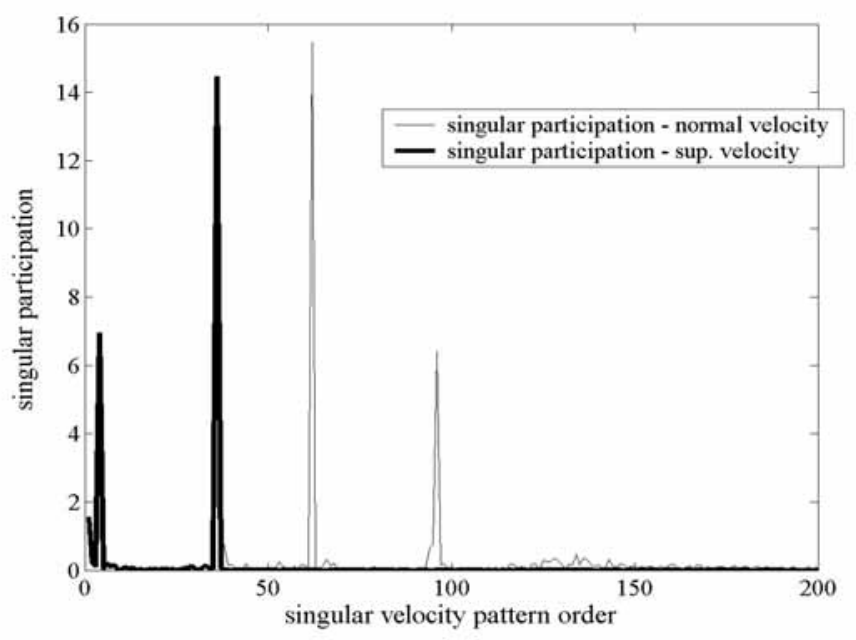

Fig. 10. Singular participation of the normal velocity and supersonic velocity for the flat-capped cylinder.

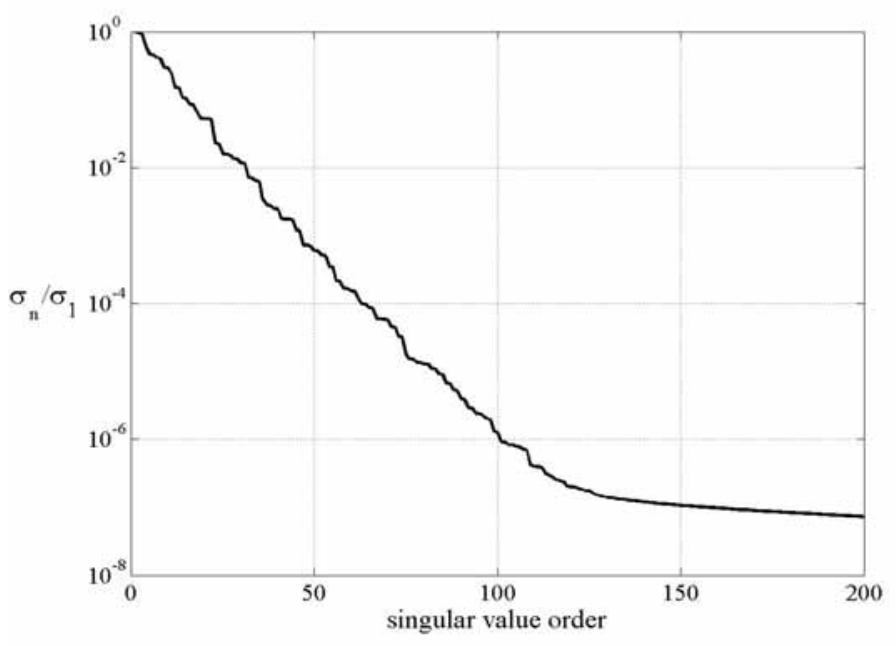

Fig. 11. Singular values decay curve for the flat-capped cylinder at $\nu=0.5$.

means, its contribution is about $30 \mathrm{~dB}$ smaller than that of the first peak. Since the third and fourth peaks are related to even smaller singular values, their contribution can be totally neglected.

Figures 14 to 16 show the results of the study conducted for the engine-shaped source. Some interesting differences can be noticed. The first one is related to the sound power convergence curve which, for the cylindrical source, reaches its final value with less than ten singular velocity patterns taken into account. For the engine-shaped source, however, this curve stabilizes only after around fifty velocity patterns are introduced. This difference is justified by the fact that, in the second case, the singular participation shows that the normal velocity has relatively low content of low order velocity patterns, causing these patterns, despite having high radiation efficiency, to have low contribution to sound power. The second difference is related to the singular participation curve. It can be clearly noticed, by comparing Figs 10 and 16, that the first is null except at the peaks, while the second presents a significantly more irregular behavior. 


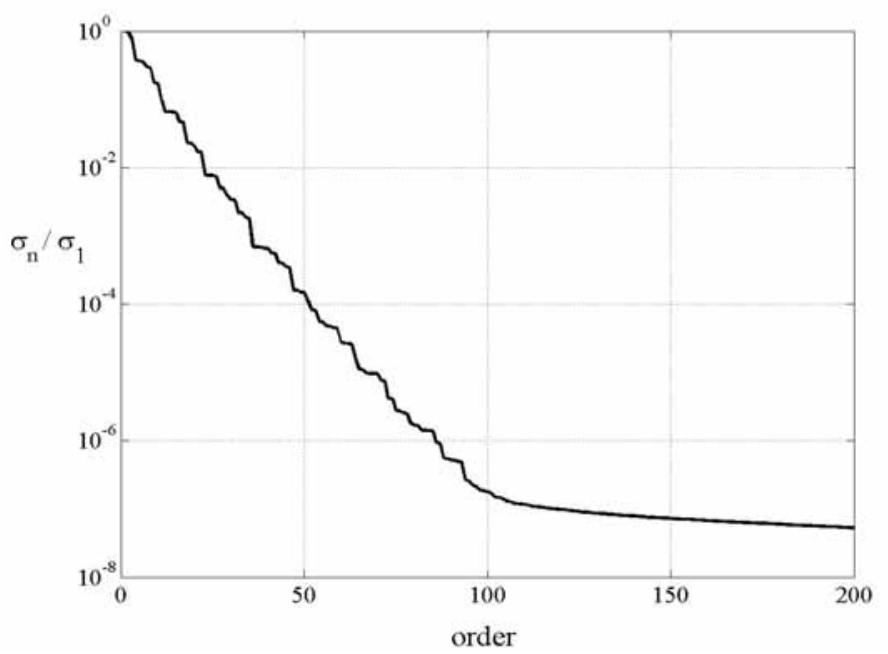

Fig. 12. Typical singular values decay curve.

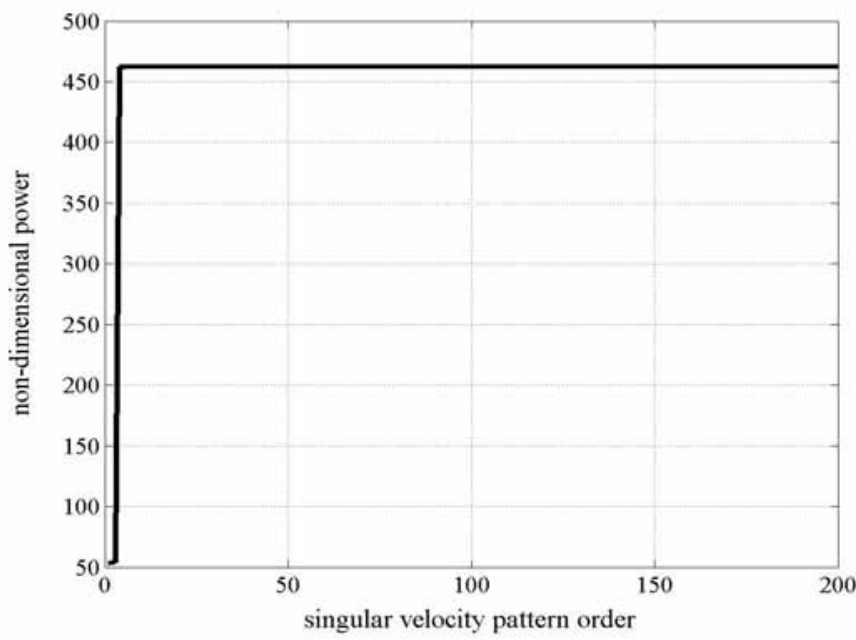

Fig. 13. Sound power convergence curve for the flat-capped cylinder.

\section{Conclusions}

The numerical results confirm, as expected, the convergence of the supersonic intensity to the active intensity as the frequency approaches coincidence, since adjacent opposite-phased anti-nodes cease to interfere destructively. The results show that the generalized supersonic intensity is capable of identifying edge modes on the flat-capped cylinder, eliminating those regions where the active intensity could mistakenly indicate a spot as being a significant contributor to the sound power. For the source roughly shaped like an internal combustion engine, the supersonic intensity was also capable of identifying the regions with high contribution to the sound power, in opposition to the conventional active intensity, which showed high values where, in fact, recirculation was taking place due to canceling.

The discrepancy between singular velocity patterns and normal vibration modes is also dealt with by analyzing the singular participation curves. The numerical results show that the more irregular the source shape, the higher the discrepancy between them will be. This fact shows that a simple analysis of the normal vibration modes may, 


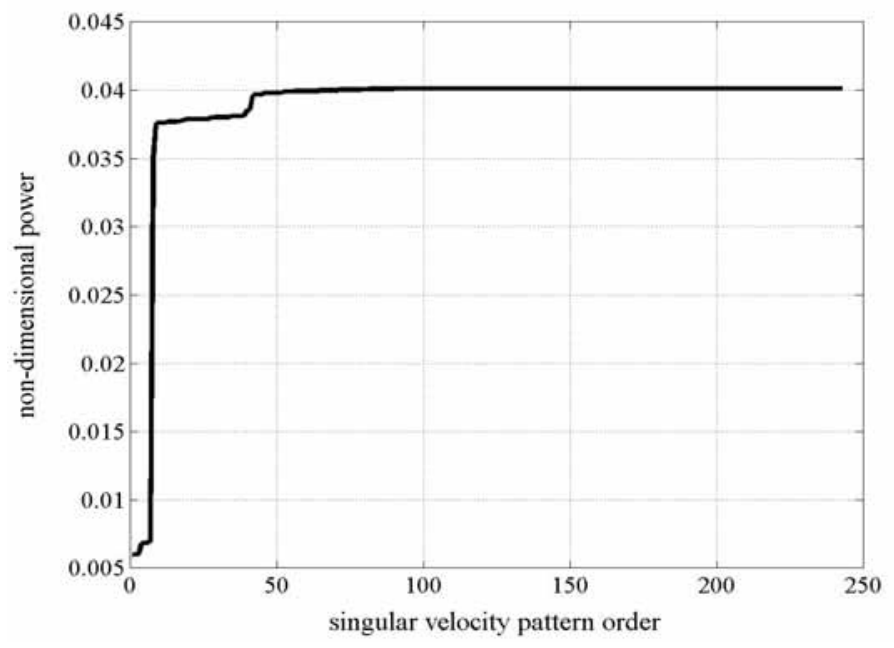

Fig. 14. Sound power convergence for the engine-shaped source.

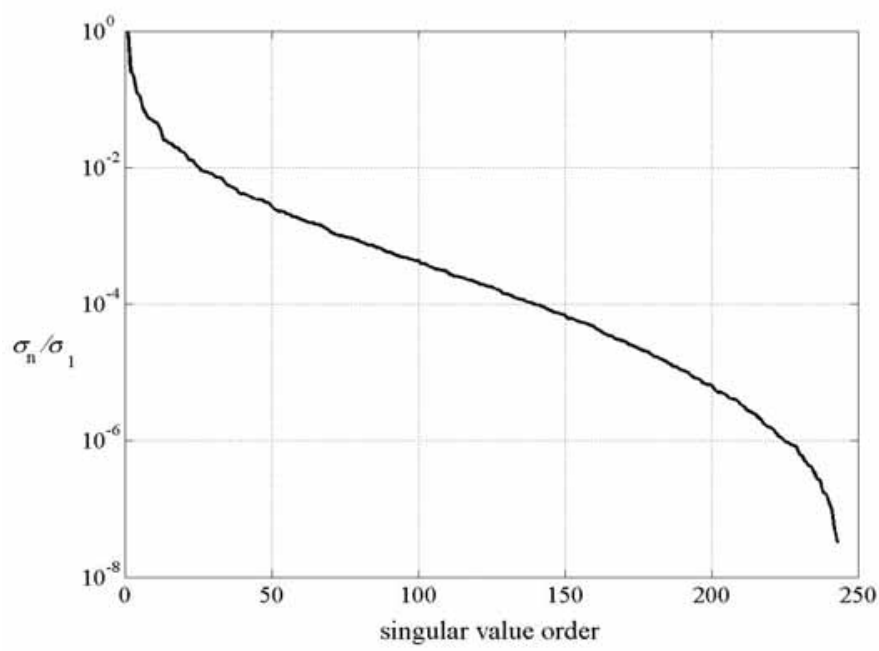

Fig. 15. Singular values decay curve for the engine-shaped source.

depending on the source geometry, not be enough for obtaining a meaningful insight about its sound radiation properties. In those cases, the SVD can be quite useful.

It was also pointed out that there is a decrease in the resolution of the supersonic intensity results due to the discarding the singular velocity patterns with low radiation efficiency, which normally present a short spatial variation (small wavelength) and are therefore responsible for the sharp spatial variation that may exist in the normal velocity distribution. This resolution loss, however, is not a limitation of the methodology, but, instead, reflects the fact that components with small wavelength present, in many cases, negligible contribution to the sound power and should, therefore, be discarded during the evaluation of the supersonic intensity.

There is a significant difference between the Fourier approach and the SVD-based one. While in the former the function which form the basis onto which the normal velocity is projected are frequency independent, in the latter it is necessary to reevaluate the matrices $\mathbf{U}, \Sigma$ and $\mathbf{V}$ whenever the frequency changes, causing the computational cost to be significantly higher in the SVD-based approach than in the Fourier-based one. This disadvantage, however, is overcomed by the possibility of broader applicability of the latter. 


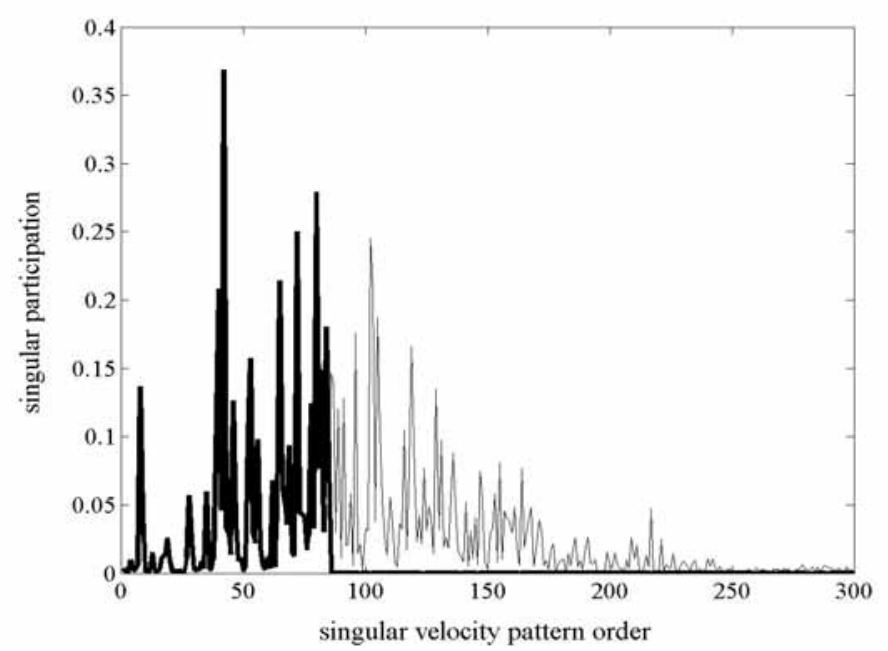

Fig. 16. Singular participation curve for the engine-shaped source.

In a general way, the obtained results show that the generalized supersonic intensity can be an auxiliary parameter in the design of potentially noisy products such as household appliances, combustion engines etc. The association of the finite elements method for simulating the source vibrational behavior with the boundary elements method, for prediction of its acoustic performance, has shown to be useful to identify, by evaluating the generalized supersonic intensity, the regions where a geometry or local stiffness change might have a greater effect on the sound power, contributing therefore to reduce it.

\section{References}

[1] G.V. Borgiotti, The power radiated by a vibrating body in an acoustic fluid and its determination from boundary measurements, J. Acoust, Soc. Am 88(4) (1990), 1884-1893.

[2] G.V. Borgiotti and K.E. Jones, The determination of the acoustic far field of a radiating body in an acoustic fluid from boundary measurements, J. Acoust, Soc. Am 93(5) (1993), 2788-2797.

[3] G.V. Borgiotti and E.M. Rosen, The determination of the far field of an acoustic radiator from sparse measurement samples in the near field, J. Acoust, Soc. Am 92(2) (1992), 807-818.

[4] R.D. Ciskowski and C.A. Brebbia, Boundary Elements Methods in Acoustics, Elsevier Applied Science, New York.

[5] G.H. Golub and C.F. Van Loan, Matrix Computations, Johns Hopkins University Press, Baltimore, MD, 1996.

[6] M.B.S. Magalhães, "Intensidade supersônica e eficiência de radiação vibroacústica de fontes com geometria arbitrária (in portuguese)", $\mathrm{PhD}$ thesis, Federal University of Rio de Janeiro, Rio de Janeiro, RJ, Brazil.

[7] M.B.S. Magalhães, R.A. Tenenbaum and M. Zindeluk, Supersonic acoustic intensity for arbitrarily shaped sources, in Proceedings of the International Congress on Acoustics 2001, Rome.

[8] G. Maidanik, Response of ribbed panels to reverberant acoustic fields, J. Acoust, Soc. Am 34(1) (1962), 809-826.

[9] J.D. Maynard, E.G. Williams and Y. Lee, Nearfield acoustic holography: I. theory of generalized holography and the development of NAH, J. Acoust, Soc. Am 78 (1985), 1395-1413.

[10] P.M. Morse and K.U. Ingard, Theoretical Acoustics, McGraw-Hill, New York, 1968.

[11] D.M. Photiadis, The relationship of singular value decomposition to wave-vector filtering in sound radiation problems, J. Acoust, Soc. Am 88(2) (1990), 1152-1159.

[12] A.D. Pierce, Acoustics: an introduction to its physical principles and applications, Acoustical Society of America, Woodbury, New York, 1991.

[13] A. Sarkissian, Reconstruction of the surface acoustic field on radiating structures, J. Acoust, Soc. Am 92(2) (1992), 825-830.

[14] P.R. Stepanishen and K.C. Benjamin, Forward and backward projection of acoustic fields using FFT methods, J. Acoust, Soc. Am 71(4) (1982), 803-812.

[15] W.A. Veronesi and J.D. Maynard, Digital holographic reconstruction of sources with arbitrarily shaped surfaces, J. Acoust, Soc. Am 85 (1989), 588-598.

[16] E.G. Williams, Supersonic acoustic intensity, J. Acoust, Soc. Am 97(1) (1995), 121-127.

[17] E.G. Williams, Supersonic acoustic intensity on planar sources, J. Acoust, Soc. Am 104(5) (1998), $2845-2850$.

[18] E.G. Williams, Fourier Acoustics - sound radiation and nearfield acoustical holography, Academic Press, London, 1999. 
[19] E.G. Williams, Methods to localize radiated noise sources on structures, in Proceedings of NOVEM 2000, $2000,1-8$.

[20] E.G. Williams, Supersonic acoustic intensity, Private Comunication, 2002.

[21] T.W. Wu, (editor), Boundary elements in acoustic - fundamentals and computer codes, WIT Press, Southampon, UK, 2000. 

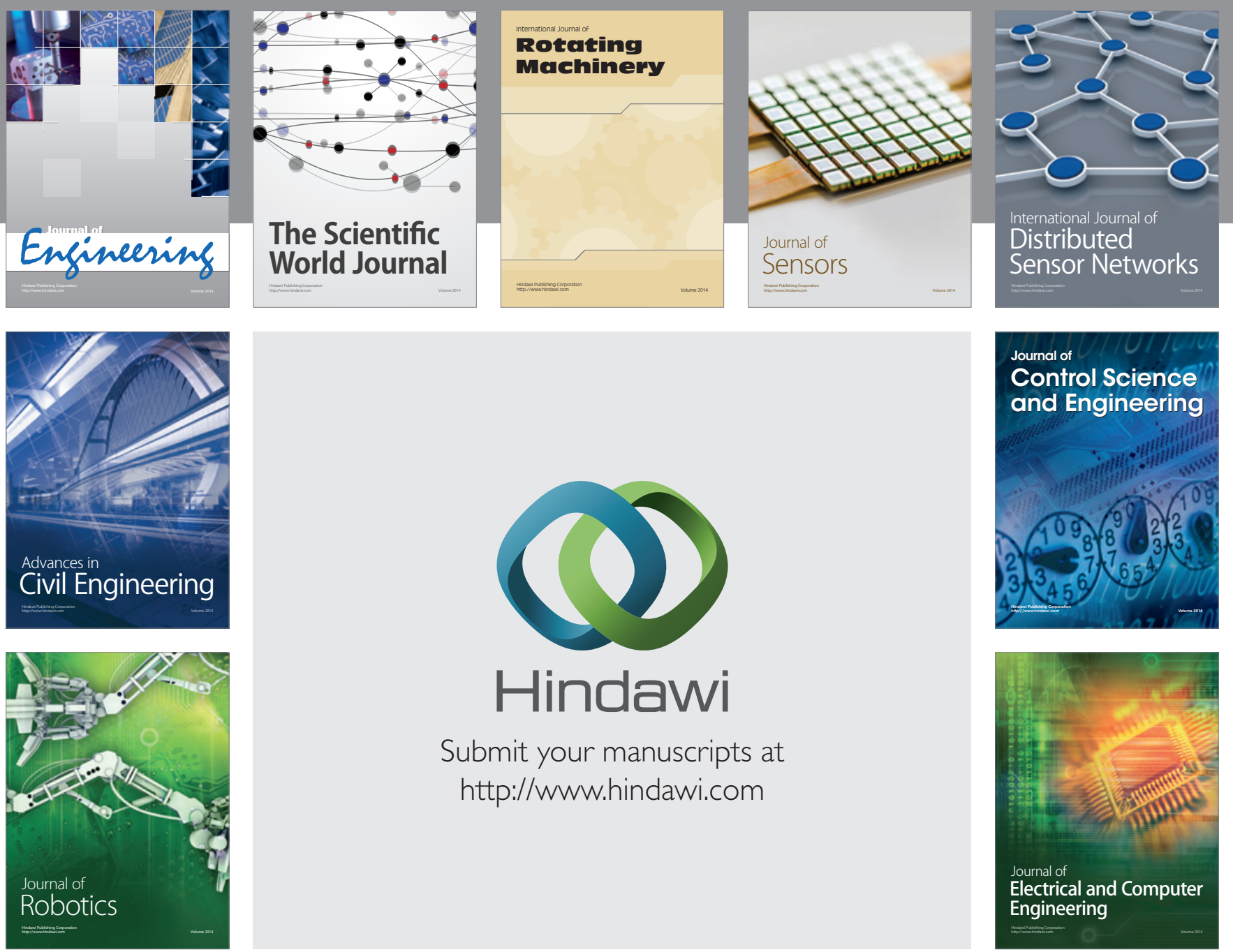

Submit your manuscripts at

http://www.hindawi.com
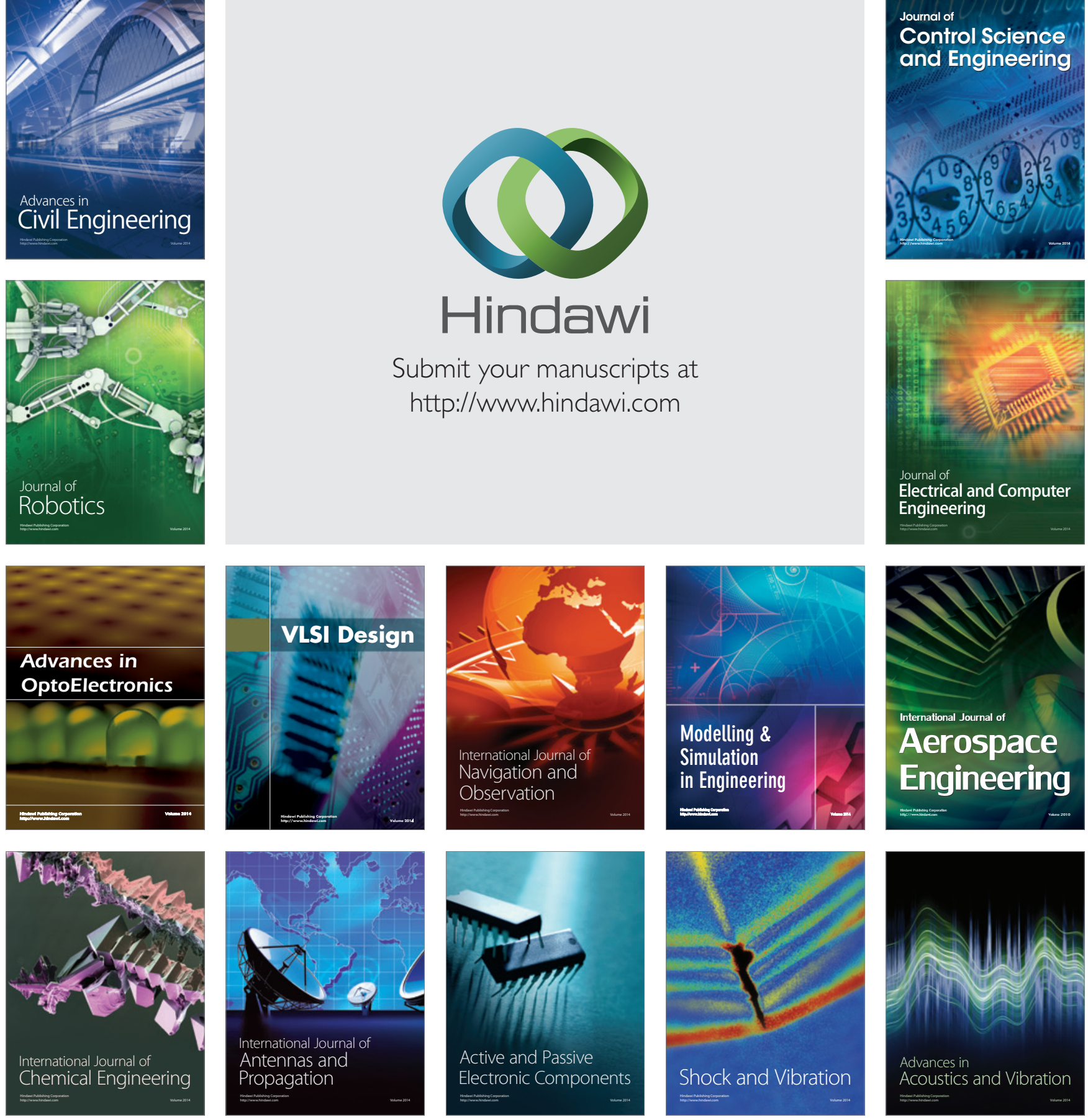